\title{
Comparison of electrically elicited responses in rabbit and mouse retinal ganglion cells
}

\author{
Paul Werginz, Member, IEEE, and Shelley I. Fried, Member, IEEE
}

\begin{abstract}
Retinal implants are currently the only commercially available devices that can restore vision in patients suffering from a wide range of outer retinal degenerations. In order to improve the clinical outcome, i.e. the quality of elicited vision, a large number of in-vitro experiments probing the impact of electric stimulation on activation of the retina have been conducted. In these studies, however, retinas from many different species have been used which impedes comparisons between studies. Therefore, we measured the responses from four major ganglion cell types to light and electric stimulation in rabbit and mouse retina and compared their responses. We found strong similarities between the two species in transient cells whereas responses in sustained cell types typically did not match as well.
\end{abstract}

\section{INTRODUCTION}

A large number of different retinal ganglion cell (RGC) types in the mammalian retina have been reported $[1,2]$. Mostly, data in response to light stimulation (LS) was obtained and compared across species. Thereby, cells with similar responses to LS were suggested to be (functional) homologs, however, no detailed comparison studies are available.

The development of new stimulation strategies for retinal implants requires a more detailed knowledge of how electric stimulation (ES) activates distinct cell types within the retina. Importantly, the utility of results from lower order species such as mice is limited without the knowledge which cell types respond similarly to ES (and LS), i.e. which cell types are electrically homologous. We showed previously that different RGC types in rabbit retina exhibit distinct responses to both LS and ES [3,4]. In the current study we compare responses of these RGCs to light responses from potential homologs in mouse retina and subsequently explore their responses to ES as well.

\section{METHODS}

The care and use of animals followed all federal and institutional guidelines and all protocols were approved by

*Research was supported by the Austrian Science Fund (FWF, J3947), by the VA (RR\&D: RX001663) and NIH (U01-NS099700). Both authors were with the Department of Neurosurgery, Massachusetts General Hospital, Harvard Medical School, Boston, MA 02114 USA at the time of this study

P. Werginz is also with the Institute for Analysis and Scientific Computing, Vienna University of Technology, 1040 Vienna, Austria (email: werginz.paul@mgh.harvard.edu).

S. I. Fried is also with the Boston VA Healthcare System, Rehabilitation, Research and Development, Boston, MA 01230 USA (e-mail: fried.shelley@mgh.harvard.edu). the Institutional Animal Care and Use Committee (IACUC) of the Massachusetts General Hospital.

New Zealand White rabbits (Charles River Laboratories, $\sim 2 \mathrm{~kg}$ ) were anaesthetized with a mix of ketamine (75 $\mathrm{mg} / \mathrm{kg}$, Henry Schein) and xylazine (10 mg/kg, Akorn Animal Health). Animals were subsequently euthanized by an intracardial injection of euthasol (Virbac) and the eyes were harvested. Wild-type mice (C57BL/6J, Charles River Laboratories) were anaesthetized with isofluorane (Henry Schein) and subsequently euthanized by cervical dislocation.

The retina was separated from the sclera and pigment epithelium and mounted, photoreceptor side down, onto a recording chamber using filter paper. The retina was subsequently perfused with oxygenated Ames medium (Sigma-Aldrich) at a flow rate of $2-3 \mathrm{ml} / \mathrm{min}$ for the duration of the experiment. Temperature was maintained at $\sim 34{ }^{\circ} \mathrm{C}$. In rabbits, cells were located approximately 3-5 $\mathrm{mm}$ inferior to the visual streak whereas in mice cells across the whole retina were targeted. Small holes were made in the inner limiting membrane in order to access the RGC somata. Spiking responses to light and electric stimuli were obtained using loose (cell-attached) patch recordings. Patch electrode resistance was 8-14 $\mathrm{M} \Omega$.

LS consisted of bright or dark spots with diameters ranging from $100-1000 \mu \mathrm{m}$ and presented for 1 second. ES was delivered by a $10 \mathrm{k} \Omega$ electrode (Micro-Probes) located $\sim 25 \mu \mathrm{m}$ above the inner limiting membrane and centered over the soma. The stimulus was a $4 \mathrm{~ms}$ cathodic monophasic half-sinusoid with amplitudes of 50, 80 and 100 $\mu \mathrm{A}$. We used monophasic pulses to facilitate comparisons with previous work; charge-balancing anodic phases were delivered 1 second after cathodal onset and helped to avoid electrode damage. Stimuli were delivered by a stimulus generator (STG 2004, Multi-Channel Systems MCS GmbH).

Stimulus control and data acquisition were performed with custom software written in LabView (National Instruments) and Matlab (Mathworks). Data were recorded using an Axopatch 700B amplifier (Molecular Devices) and digitized by a data acquisition card (PCI-MIO-16E-4, National Instruments). The timing of individual spikes was detected as the depolarization (negative) peak of each spike in the raw trace. The first and last spikes of a burst in response to ES were detected manually. Firing rate was computed by pooling responses from multiple trials $(\geq 3$ for $\mathrm{LS}, \geq 5$ for ES) and subsequent convolution with a $50 \mathrm{~ms}$ (LS) or $20 \mathrm{~ms}$ (ES) sliding window.

We used the two-sample t-test to compare between the two groups (mouse and rabbit). Numerical values are presented as mean \pm 1 standard deviation of mean (SD). Statistical analysis was performed in Matlab. 


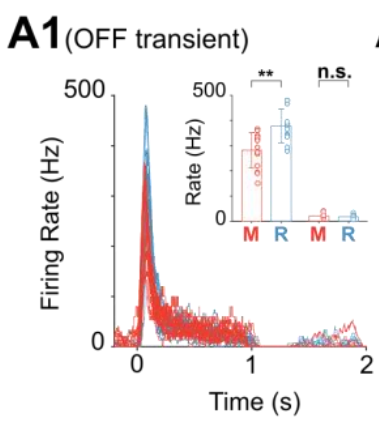

A2 (ON transient)
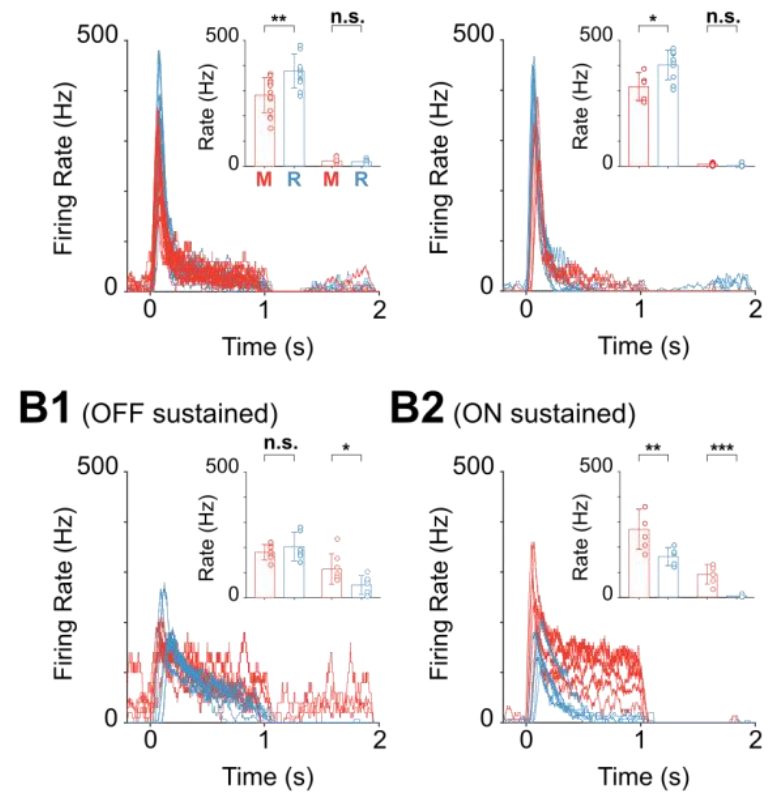

B2 (ON sustained)

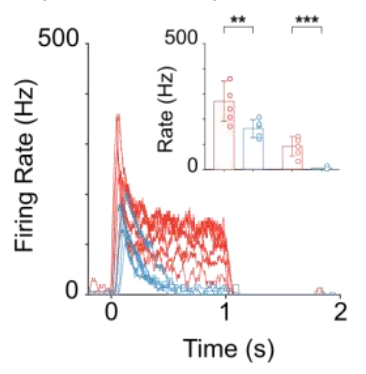

Figure 1. Responses to LS in pairs mouse and rabbit RGCs. (A) Overlays of firing rate over time of transient OFF (A1) and ON (A2) cells in mouse (red) and rabbit (blue) retina in response to a stationary light flash. Insets show a direct comparison of the average peak (left) and sustained (right) spiking rate in both species. (B) Responses to LS in sustained cells.

\section{RESUlts}

Four different cell types in mouse and rabbit retina were identified by their responses to light [4,5]. Following identification, cells were stimulated electrically and responses were compared between cells that had similar light responses, e.g. transient and sustained light responses, respectively. In total, we recorded from 33 mouse $(\mathrm{ON}-\alpha$ Transient $(\mathrm{T})=6$, OFF $-\alpha \mathrm{T}=14$, ON $-\alpha$ Sustained $(\mathrm{S})=7$, OFF$\alpha \mathrm{S}=6)$ and 33 rabbit (ON-Brisk Transient $(\mathrm{BT})=10$, OFF$\mathrm{BT}=12$, ON-Brisk Sustained (BS) $=5$, OFF-BS=6) RGCs. In this study, we use the term transient for $\alpha \mathrm{T}$ and BT cells and sustained for $\alpha \mathrm{S}$ and BS cells, respectively.

\section{A. Visual responses}

ON and OFF transient RGCs could be identified by their characteristic light response to stationary flashes (Fig. 1). Casual observation revealed that light responses in transient cells matched between mouse and rabbit (Fig. 1A); light onset or offset resulted in a strong transient response which was follow by weak sustained firing. A significant difference in peak firing rate for optimal stimulus size, i.e. the spot size that generated highest firing frequency, was observed between mouse and rabbit cells (Inset left, OFF: $p=0.0016$; $\mathrm{ON}: \mathrm{p}=0.0168)$. On the other hand, firing rate during the sustained phase, defined as the mean spiking rate during the last $200 \mathrm{~ms}$ of each response, was similar in both species (Inset right, OFF: $\mathrm{p}=0.4159$; ON: $\mathrm{p}=0.2683$ ).

In OFF sustained cells peak spiking frequency was similar across mouse and rabbit RGCs (Fig. 1B1, Inset left, $\mathrm{p}=0.3798$ ). Visual inspection, however, suggests larger differences between the light responses. Whereas mouse
OFF sustained RGCs responded with a nearly constant spiking frequency throughout the duration of the stimulation sustained cells in rabbit showed a distinct transient peak followed by a continuously decreasing firing rate (Fig. 1B1). Similarly, light responses in sustained ON RGCs in mouse and rabbit did not match well as mouse RGCs responded with significantly higher peak spiking frequencies than cells in rabbit (Fig. 1B2, Inset left, p=0.0098). Furthermore, the sustained firing rate in mouse remained at a higher level than in rabbit (Inset right, OFF: $p=0.0451$; ON: $p=9 e-4$ ).

Taken together, our results show that light responses of transient cells (Fig. 1A) in mouse and rabbit retina are more similar to each other than responses in sustained cells (Fig. 1B).

\section{B. Electric responses - Transient cells}

We next compared responses to ES in order to determine whether similarities in light responses across species also translate into similarities in their corresponding electric responses. Electric responses were evoked by a single $4 \mathrm{~ms}$ half-sinusoidal stimulus and were recorded for a 1 second period after pulse onset. In transient OFF RGCs the responses consisted of two distinct phases: i) a short burst (1-2 spikes) of direct RGC activation which is generated at the RGC axon initial segment [6] (Fig. 2A1, black arrow) and ii) a single network-mediated burst generated by activation of photoreceptors and bipolar cells (Fig. 2A1, red ellipse). Since we were mainly interested in responses elicited by the presynaptic network we only compared responses from the delayed, network-mediated burst [7]. No statistically significant difference between mouse and rabbit RGCs was found for burst duration (Fig. 2B1 left, $\mathrm{p}=0.1803$ ) or onset latency (Fig. 2B1 right, $\mathrm{p}=0.8964$ ). Similar to responses during LS, peak firing rate was significantly lower in mouse cells compared to responses from rabbit RGCs (Fig. 2B1 middle, $\mathrm{p}=2 \mathrm{e}-5$ ).

Transient ON RGCs showed a more complex response to ES in both species (Fig. 2A2). Three distinct bursts were elicited by a single stimulus: i) a short latency burst consisting of direct spikes as well as indirect spikes elicited by network activation (Fig. 2A2, black arrow); ii) a first (red circle) and iii) a second (blue ellipse) network-mediated burst. Similar to OFF transient RGCs, we only compared responses from network-mediated bursts. We analyzed burst duration, peak firing rate and onset latency for each burst separately. The duration of the first network burst was slightly longer in mouse than in rabbit RGCs (Fig. 2B2 top left, $\mathrm{p}=0.0458$ ). Peak firing rate (Fig. 2B2 top middle, $\mathrm{p}=0.3934$ ) and onset latency (Fig. 2B2 top right, $\mathrm{p}=0.1081$ ), on the other hand, were not statistically different. Properties of the latter network burst appeared to be similar in both species as average burst duration $(\mathrm{p}=0.2236)$, peak firing rate $(\mathrm{p}=0.8470)$ and onset latency $(\mathrm{p}=0.1763)$ did not show any statistically significant differences (Fig. 2B2 bottom row).

In summary, electric responses from $\mathrm{ON}$ and $\mathrm{OFF}$ transient RGCs showed strong similarities in mouse and rabbit which parallels the good correlation of their respective light responses. 
A1

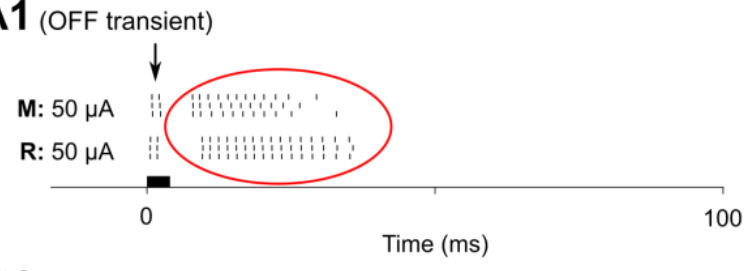

B1
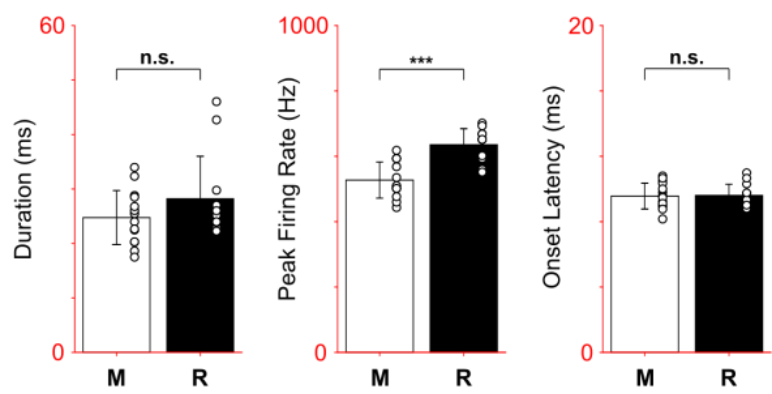

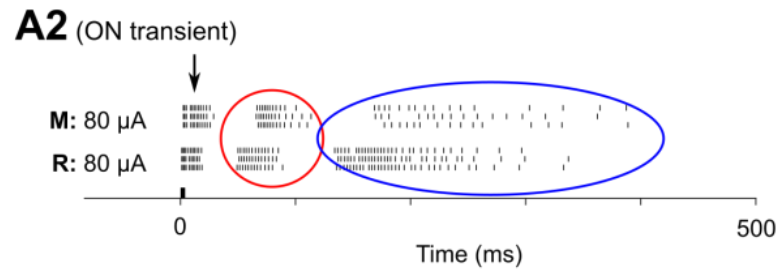

\section{B2}
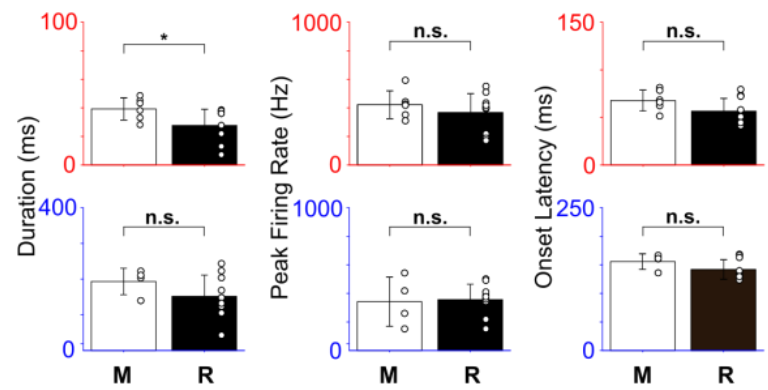

Figure 2. (A) Raster plots of transient OFF (A1) and ON (A2) mouse (top) and rabbit (bottom) RGCs in response to ES with a half-sinusoidal cathodic pulse. Stimulus amplitude was $50 \mu \mathrm{A}$ for OFF and $80 \mu \mathrm{A}$ for ON cells. Bursts that were compared are indicated by the red and blue ellipses, respectively. The timing of the $4 \mathrm{~ms}$ pulse is indicated at the bottom. (B) Comparison of three properties of each burst indicated in (A) during ES. Axis colors indicate the corresponding burst in (A).

\section{OFF TRANSIENT}

\begin{tabular}{c|c|c|c|c|c}
\hline \multicolumn{2}{c|}{ Duration (ms) } & \multicolumn{2}{c|}{ Peak Rate (Hz) } & \multicolumn{2}{c}{ Onset Latency (ms) } \\
\hline Mouse & Rabbit & Mouse & Rabbit & Mouse & Rabbit \\
\hline $24.7 \pm 5.0$ & $28.2 \pm 7.8$ & $527 \pm 55$ & $635 \pm 49$ & $9.6 \pm 0.8$ & $9.6 \pm 0.7$
\end{tabular}

ON TRANSIENT

\begin{tabular}{c|c|c|c|c|c}
\hline \multicolumn{2}{c|}{ Duration (ms) } & \multicolumn{2}{c|}{ Peak Rate (Hz) } & \multicolumn{2}{c}{ Onset Latency (ms) } \\
\hline Mouse & Rabbit & Mouse & Rabbit & Mouse & Rabbit \\
\hline $39.3 \pm 7.7$ & $27.7 \pm 11.4$ & $422 \pm 97$ & $367 \pm 132$ & $67.7 \pm 11.0$ & $56.6 \pm 13.4$ \\
\hline $193.5 \pm 37.1$ & $151.8 \pm 59.7$ & $342 \pm 173$ & $357 \pm 108$ & $155.8 \pm 13.7$ & $141.8 \pm 17.2$
\end{tabular}

OFF SUSTAINED

\begin{tabular}{c|c|c|c|c|c}
\hline \multicolumn{2}{c|}{ Duration (ms) } & \multicolumn{2}{c|}{ Peak Rate (Hz) } & \multicolumn{2}{c}{ Onset Latency (ms) } \\
\hline Mouse & Rabbit & Mouse & Rabbit & Mouse & Rabbit \\
\hline $16.1 \pm 4.4$ & $49.5 \pm 32.7$ & $305 \pm 65$ & $322 \pm 82$ & $11.3 \pm 0.8$ & $11.0 \pm 2.2$
\end{tabular}

ON SUSTAINED

\begin{tabular}{c|c|c|c|c|c}
\hline \multicolumn{2}{c|}{ Duration (ms) } & \multicolumn{2}{c|}{ Peak Rate (Hz) } & \multicolumn{2}{c}{ Onset Latency (ms) } \\
\hline Mouse & Rabbit & Mouse & Rabbit & Mouse & Rabbit \\
\hline $472.7 \pm 101$ & $295.6 \pm 43.8$ & $272 \pm 89$ & $159 \pm 35$ & $165.2 \pm 16.5$ & $167.4 \pm 14.5$
\end{tabular}

Table 1. Summary of average burst duration, peak firing rate and onset latency for network-mediated bursts in all four cell types in both species. For ON transient cells parameters from both analyzed bursts are shown.

\section{Electric responses - Sustained cells}

Similar to our approach with transient cells, we also compared responses between sustained RGCs of mouse and rabbit retinas (Fig. 3). Again, we only compared responses from bursts arising through activation of the retinal network (Fig. 3A, red ellipses). Mouse OFF sustained cells had rather short network-driven responses to ES whereas sustained cells in rabbit responded with significantly longer spike trains to the same stimulus (Fig. 3B1 left, $\mathrm{p}=0.0208$ ). Peak firing rate (Fig. 3B1 middle, $\mathrm{p}=0.6775$ ) and onset latency (Fig. 3B1 right, $\mathrm{p}=0.7352$ ) were similar for the two groups.
Since the thresholds for eliciting robust network-mediated responses were different for ON sustained RGCs in mouse versus those in rabbit RGCs, we used two different amplitudes for comparison. Mouse ON sustained cells were stimulated with a higher stimulus amplitude than rabbit RGCs (100 vs. $80 \mu \mathrm{A})$. Responses typically lasted for several hundred milliseconds with longer-duration responses in rabbit RGCs (Fig. 3B2 left, p=0.0068). Additionally, peak firing rate was significantly higher in mouse RGCs (Fig. 3B2 middle, $\mathrm{p}=0.0299$ ) whereas onset latency was similar across species (Fig. 3B2 right, $\mathrm{p}=0.8286$ ).

Overall, the electric responses observed in sustained cells were less consistent across species than responses from transient RGCs.

\section{CONCLUSION}

We found that the previously reported (electric) response differences between $\mathrm{ON}$ and $\mathrm{OFF}$ cells in rabbit retina, i.e. short-latency bursts in OFF cells vs. medium- and longlatency bursts in ON cells [3,4], were also present in $\alpha$ cells of the mouse. Furthermore, the striking similarities during both, LS and ES in ON and OFF transient cells are suggesting that mouse $\alpha \mathrm{T}$ and rabbit BT RGCs are indeed functionally similar and therefore can be considered homolog. Even more surprising than similar responses to LS was the consistency of the complex electric response pattern with two/three bursts at various onset latencies was well matched across species (Fig. 2B). Further evidence for potential homology are i) large soma size, ii) large dendritic tree diameter and iii) weak inhibitory surround (data not shown). Since rabbit BT cells are thought to be homologs of parasol cells in non-human primates our results suggest that responses from mouse $\alpha \mathrm{T}$ also might be similar to responses from higher-order species. These cells therefore might be an ideal target for developing better stimulation strategies in mouse which could be applied in future retinal implants. 


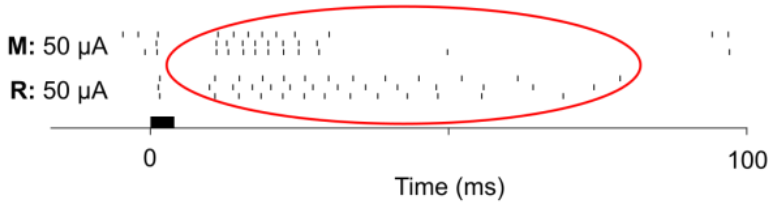

B1
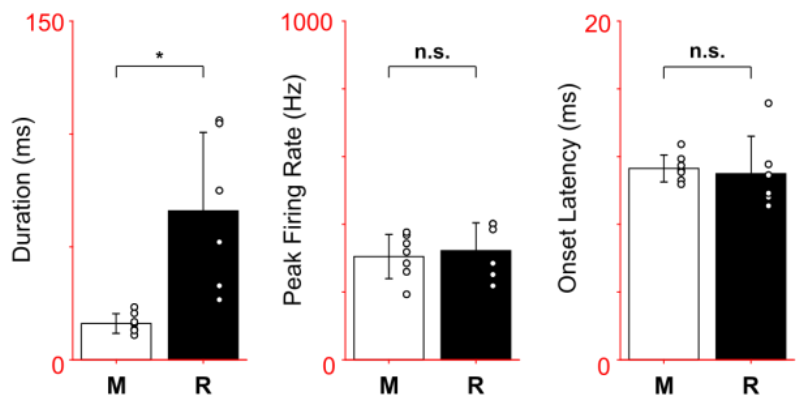

Figure 3. (A) Raster plots of sustained OFF (A1) and ON (A2) mouse (top) and rabbit (bottom) RGCs in response to ES. Stimulus amplitude was $50 \mu \mathrm{A}$ for $\mathrm{OFF}$ and 100 (mouse) and 80 (rabbit) $\mu \mathrm{A}$ for ON cells (see text). Bursts that were compared are indicated by red ellipses. The timing of the 4 ms pulse is indicated at the bottom. (B) Comparison of three properties of the bursts indicated in (A) during ES. Axis colors indicate the corresponding burst in (A).

Comparison of $\mathrm{ON}$ and $\mathrm{OFF}$ sustained RGCs across mouse and rabbit retina revealed more variations between the two species. Both, LS and ES elicited responses that were in general agreement; however, detailed analysis of various parameters indicates larger differences than in the transient population. Aside from the variations in light responses shown in Figure 1 we also observed differences in the strength of inhibitory surround (not shown). Whereas sustained RGCs in rabbit had a strong inhibitory surround preventing spiking when stimulated with large spots mouse RGCs only weakly altered their responses with increasing spot sizes $>500 \mu \mathrm{m}$. Furthermore, soma size for rabbit BS cells is in the overall mid-range whereas mouse $\alpha \mathrm{S}$ cells are the largest cells in the mouse retina [5]. Rabbit BS cells are potential homologs of midget cells in non-human primate retina and are thought to be involved in high acuity vision in the central retina. The lack of such a region of high acuity in mouse retina suggests that mouse $\alpha \mathrm{S}$ cells are not likely to be homologs of these cells.

A comparison of electric responses revealed another interesting detail. The general responses characteristics in ON cells appeared to be different between transient and sustained cells, respectively (cf. Fig. 2B and 3B). However, the onset latency of the second burst in sustained cells and the third burst in transient cells was highly similar ( 150 ms) which suggest that the presynaptic network has at least in part a similar architecture. However, the additional burst as well as a stronger first burst indicates differential activation of the sustained and transient pathway, respectively. By blockage of photoreceptor inputs we found that mediumand long-latency network bursts were mediated by photoreceptor input (data not shown). The network-driven spikes in the first burst in ON transient cells however still persisted during blockage. Therefore, it may be possible that stronger activation in ON transient cells is mediated by bipolar cell activation. Since bipolar cells are preserved in degenerate retina this also suggests that $\mathrm{ON}$ transient (parasol) cells are a potential target for stimulation with retinal implants.

\section{ACKNOWLEDGMENT}

We would like to thank Jae-Ik Lee and Vineeth Raghuram for helpful discussions during the preparation of the manuscript.

\section{REFERENCES}

[1] J. H. Caldwell and N. W. Daw, "New properties of rabbit retinal ganglion cells", J. Physiol., vol. 276, pp. 257-276, 1978.

[2] R. L. Rockhill, F. J. Daly, M. A. MacNeil, S. P. Brown and R. H. Masland, "The diversity of ganglion cells in a mammalian retina", $J$. Neurosci., vol 22, pp. 3831-3843, 2002.

[3] M. Im and S. I. Fried, "Indirect activation elicits strong correlations between light and electric responses in ON but not OFF retinal ganglion cells", J. Physiol., vol. 593, pp. 3577-3596, 2015.

[4] P. Werginz, M. Im, A. E. Hadjinicolaou and S. I. Fried, "Visual and electric spiking responses of seven types of rabbit retinal ganglion cells", Conf Proc IEEE Eng Med Biol Soc., 2434-2437, 2018.

[5] B. Krieger, M. Qiao, D. L. Rousso, J. R. Sanes and M. Meister, "Four alpha ganglion cell types in mouse retina: Function, structure, and molecular signatures", PLoS One, vol. 12, e0180091, 2017.

[6] S. I. Fried, H. A. Hsueh and F. S. Werblin, "A method for generating precise temporal patterns of retinal spiking using prosthetic stimulation", J Neurophysiol., vol. 95, pp. 970-978, 2006.

[7] M. Im and S. I. Fried, "Temporal properties of network-mediated responses to repetitive stimuli are dependent upon retinal ganglion cell type", J Neural Eng., vol. 13, 025002, 2016.

(C) 2019 IEEE. Personal use of this material is permitted. Permission from IEEE must be obtained for all other uses, in any current or future media, including reprinting/republishing this material for advertising or promotional purposes, creating new collective works, for resale or redistribution to servers or lists, or reuse of any copyrighted component of this work in other works. 